\title{
Multiple recurrent postoperative spinal infections due to an unrecognized presacral abscess following placement of bicortical sacral screws: case report
}

\author{
Laura Bloom, BA, S. Shelby Burks, MD, and Allan D. Levi, MD, PhD \\ Department of Neurological Surgery and The Miami Project to Cure Paralysis, University of Miami Miller School of Medicine, \\ Miami, Florida \\ Postoperative wound infections in spinal surgery remain an important complication to diagnose and treat successfully. \\ In most cases of deep infection, even with instrumentation, aggressive soft-tissue debridement followed by intravenous \\ antibiotics is sufficient. This report presents a patient who underwent L3-S1 laminectomy and pedicle screw placement \\ including bicortical sacral screws. This patient went on to develop multiple (7) recurrent infections at the operative site \\ over a 5-year period. Continued investigation eventually revealed a large presacral abscess, which remained the source \\ of recurrent bacterial seeding via the remaining bone tracts of the bicortical sacral screws placed during the original \\ lumbar surgery. Two years after drainage of this presacral collection via a retroperitoneal approach, the patient remains \\ symptom free.
}

http://thejns.org/doi/abs/10.3171/2015.4.SPINE141059

KEY WORDS instrumentation; methicillin-resistant Staphylococcus aureus; paraspinal and presacral; postoperative infection; spine

$\mathrm{S}$ URGICAL site infections (SSIs) are a relatively common complication following spinal surgery, with an incidence of $0.2 \%-12 \%$, depending on important factors such as open versus minimally invasive procedure and whether spinal instrumentation is used..$^{4,14,16}$ Treatment for deep-seated infections typically consists of an incision and drainage (I \& D), removal of devitalized and infected tissues, and wound drains followed by intravenous (IV) antibiotics. In nearly all cases this is sufficient to successfully treat the infection. ${ }^{3,6,11,14,18}$ Even with recurrences it is very rare to require more than 2 I \& Ds, and in those resistant cases where secondary osteomyelitis develops, removal of the instrumentation is all that is required to cure the patient in the vast majority of cases. . $^{3,6,11,14,18}$ Here we present a case of multiple recurrent infections over a 5-year period after lumbar spinal surgery-despite 6 prior I \& Ds and prolonged treatment with broad-spectrum and then suppressive antibiotics before the patient received the correct diagnosis and definitive treatment requiring both anterior presacral and posterior drainage.

\section{Case Report}

A 61-year-old man, a nonsmoker with a medical history of hypertension and asthma, originally presented with intractable low-back pain and leg pain on the right side in an L-4 distribution. Admission CT and MRI scans revealed an L3-S1 spondylosis with multilevel lateral recess stenosis. The patient underwent laminectomies from L-3 to $\mathrm{S}-1$, with bilateral facetectomies and foraminotomies from L-3 to S-1. Pedicle screws were placed bilaterally from L-3 to S-1 with bicortical sacral screws (Fig. 1A). Local bone, supplemented with morselized bone allograft and sponges soaked with recombinant human bone morphogenetic 

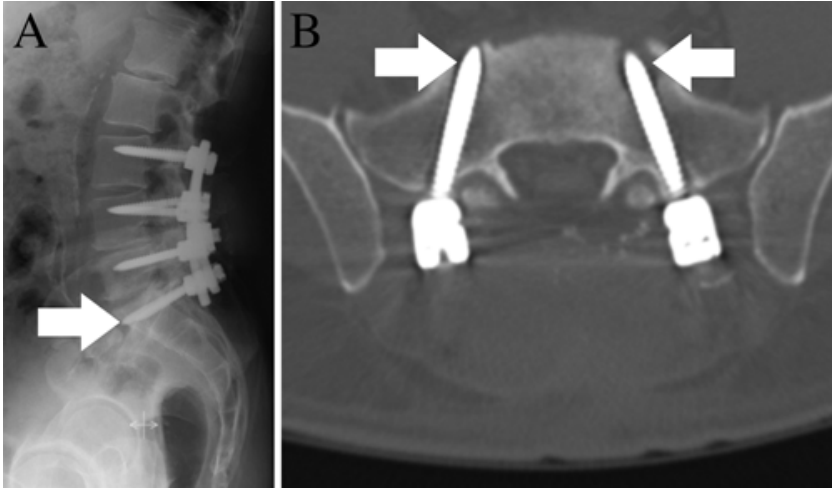

FIG. 1. A: Lateral radiograph depicting lumbar instrumentation obtained shortly after original surgery. The bicortical S-1 screws pass through the anterior surface of the S-1 vertebra (arrow). B: Axial CT scan at S-1 level taken 6 months postoperatively. Bicortical location can again be noted as well as a degree of lucency around both pedicle screws (arrows).

protein-2 (Medtronic, Inc.), was implanted posterolaterally along the construct. On the 7th day after his lumbar surgery the patient returned home.

Approximately 1 month later the patient returned to clinic reporting fevers and chills as well as drainage from his lumbar incision. He was admitted for suspected infection, and a Gram stain of aspirated fluid showed grampositive cocci. The patient underwent I \& D of a deep lumbar wound infection. Cultures demonstrated growth of methicillin-resistant Staphylococcus aureus (MRSA). The patient was discharged on postoperative Day 5 with an 8 -week course of IV vancomycin.

Approximately 9 months after his first I \& D, the patient developed a recurrent deep-seated infection, which was managed with a repeat (second) I \& D and removal of all hardware (Fig. 1B). A course of IV antibiotics was completed. Despite these interventions the patient went on to require 4 more I \& Ds over a 30-month period. These recurrences were treated similarly with I \& D and longterm IV antibiotics, followed by 1 year of suppressive therapy with doxycycline.

Five years after the original surgery, the patient presented to our hospital with his seventh SSI. On this occasion MRI and CT scans noted the presence of multiple paraspinal collections posteriorly and laterally on the left. The MRI scan also detected the presence of a presacral collection extending from L-5 to S-5. Evidence of an infection in this presacral and posterior paraspinal location necessitated both anterior and posterolateral approaches to adequately debride the infectious material (Fig. 2). The surgical procedure consisted of the following: 1) a left retroperitoneal approach to drain the presacral abscess, which drained $100 \mathrm{ml}$ of pus under high pressure; and 2) a posterior approach to drain the posterolateral abscess on the left, with drains placed in both locations. Cultures grew MRSA and the patient was then placed on an 8-week course of IV daptomycin and Zosyn. Postoperative MRI scans demonstrated marked reduction in the size of the abscess after 3 months. The patient was restarted on suppressive oral antibiotic therapy (doxycycline), to be contin-
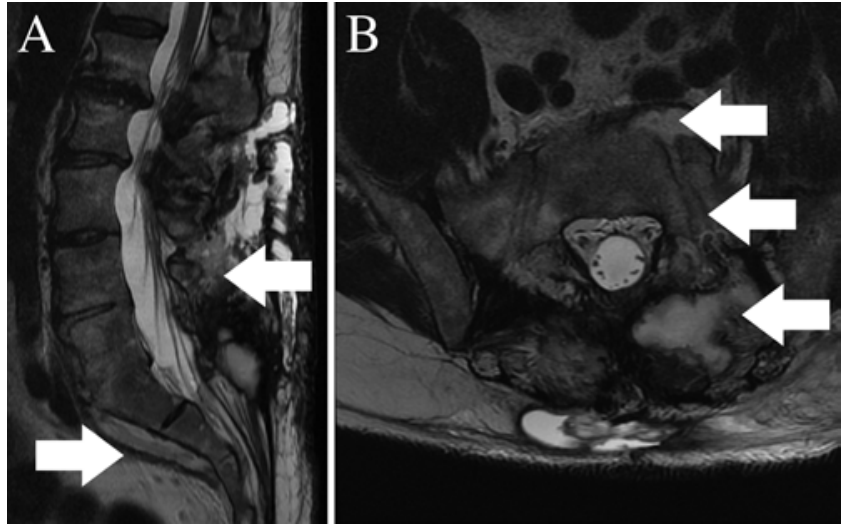

FIG. 2. A: Sagittal T2-weighted scan demonstrating extensive collection in paraspinal, superficial, and presacral locations (arrows). This MRI scan was taken just before the combined anterior-posterior debridement preformed at our institution, approximately 5 years after the original lumbar surgery. B: Axial T2-weighted scan demonstrating the communication of infectious material through the S-1 screw tract on the left (arrows).

ued indefinitely. The patient has been followed closely for 2 years, and to date the presacral and paraspinal collections remain minimal and the patient has not had recurrent symptoms to suggest infection (Fig. 3).

\section{Discussion}

The standard of care in treating SSIs presenting as deep infections after spinal instrumentation consists of I \& D followed by IV antibiotics. Early and aggressive handling of the infected soft tissues typically allows the patient to retain the hardware. ${ }^{2,3,6,8,14,19}$ There have been no reports of multiple recurrences from a presacral source.

Surgical site infection represents a significant challenge following lumbar spinal surgery-and in open procedures with a posterior approach in the presence of instrumentation even more so. Postoperative spinal infection rates range from $0.2 \%$ to $12 \%$, with posterior instrumented procedures-particularly in the setting of cancer-falling in the higher end of this range., ${ }^{3,12,14,16}$ Surgical spinal procedures and the use of instrumentation are increasing each year, and therefore managing postoperative infections is becoming increasingly important.

Although all lumbar spinal surgeries carry a risk of infection, several factors increase this risk, including the following: posterior surgical approach, open surgical procedure, and the presence of instrumentation. . $^{3,7,7,8,10,12,14,15,19}$ Additionally, the presence of instrumentation can make infections more difficult to treat ${ }^{14,15,19}$ Longer operating times and prolonged retraction associated with the placement of instrumentation probably contribute. ${ }^{17,19}$ Although the literature is not unanimous, several studies cite the benefit of retaining spinal implants in the treatment of acute SSI. Quaile states that the aim is to retain implants if possible, ${ }^{19}$ and Levi et al. advocate leaving hardware in place whenever feasible, so long as it is securely attached to the spine. In a retrospective study, hardware was maintained in 16 of 17 successfully treated SSIs. ${ }^{14}$ Once the SSI becomes chronic, several studies advocate removal of 


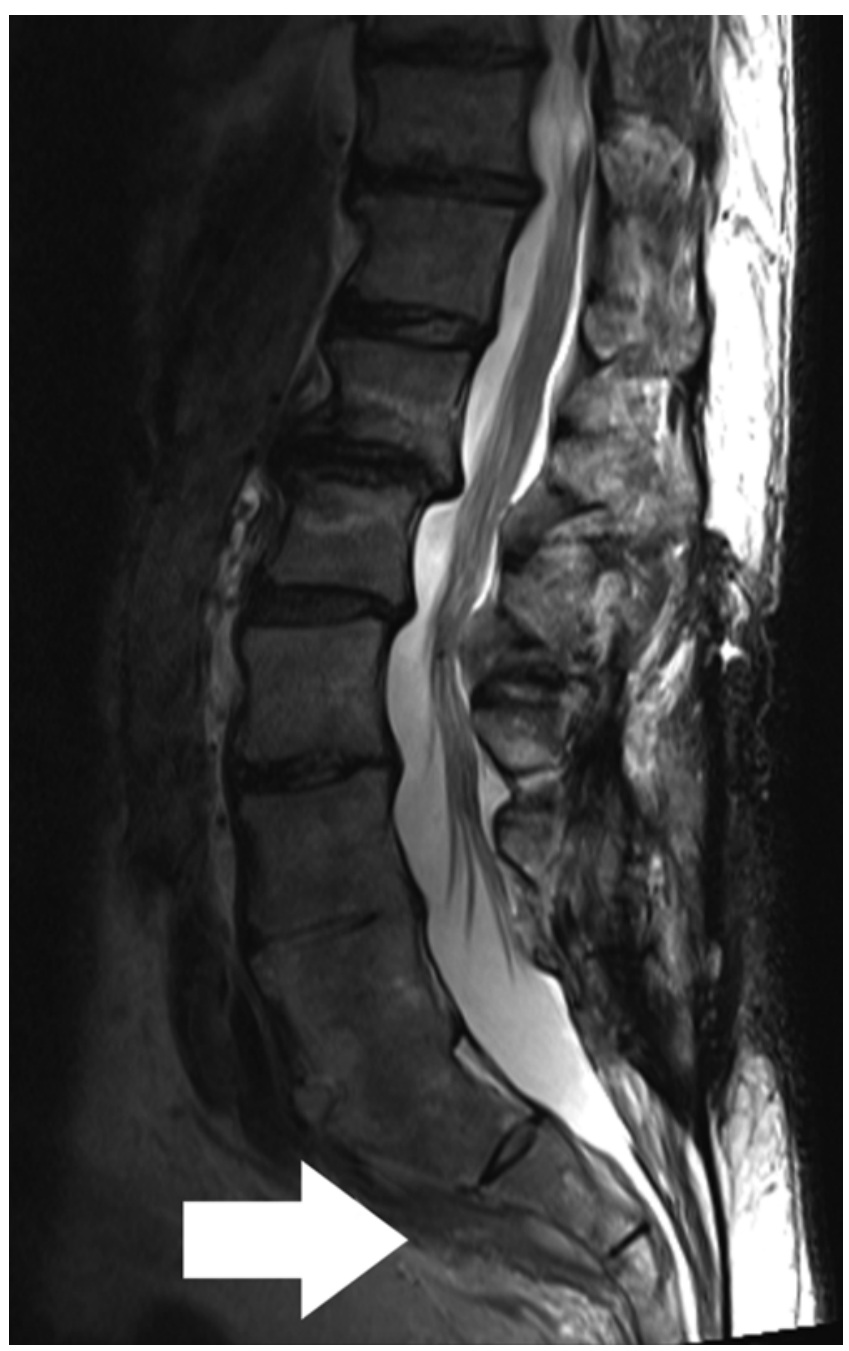

FIG. 3. Sagittal T2-weighted MRI scan taken at 8 months after combined anterior-posterior debridement demonstrates a significant reduction in the presacral collection (arrow).

instrumentation, because retention is associated with persistent and recurrent infections. ${ }^{2,6,9}$

Although instrumentation does markedly increase the risk of infection, it is often necessary when the patient's spine requires stabilization. ${ }^{1,14,19,20}$ Bicortical screws are a good example of this: by breaching 2 segments of cortical bone, these screws offer a greater degree of stability than do monocortical screws. ${ }^{5,13,21}$ This is especially important at the lumbosacral junction when a long fusion lever arm is present. ${ }^{13}$ On the other hand, our patient's case shows that as a screw passes through the anterior surface of the vertebrae it opens the potential for reinfection through recurrent seeding of the sacral tract. Similarly, if large-diameter screws are used and bicortical penetration is achieved, one will create a larger lumen for the transfer of material and fluid between the posterior and anterior aspects of the vertebral column. This is most relevant in the setting of infection, and thus must be considered. On retrospective review of imaging that was performed at another hospital approximately 18 months after removal of hardware,

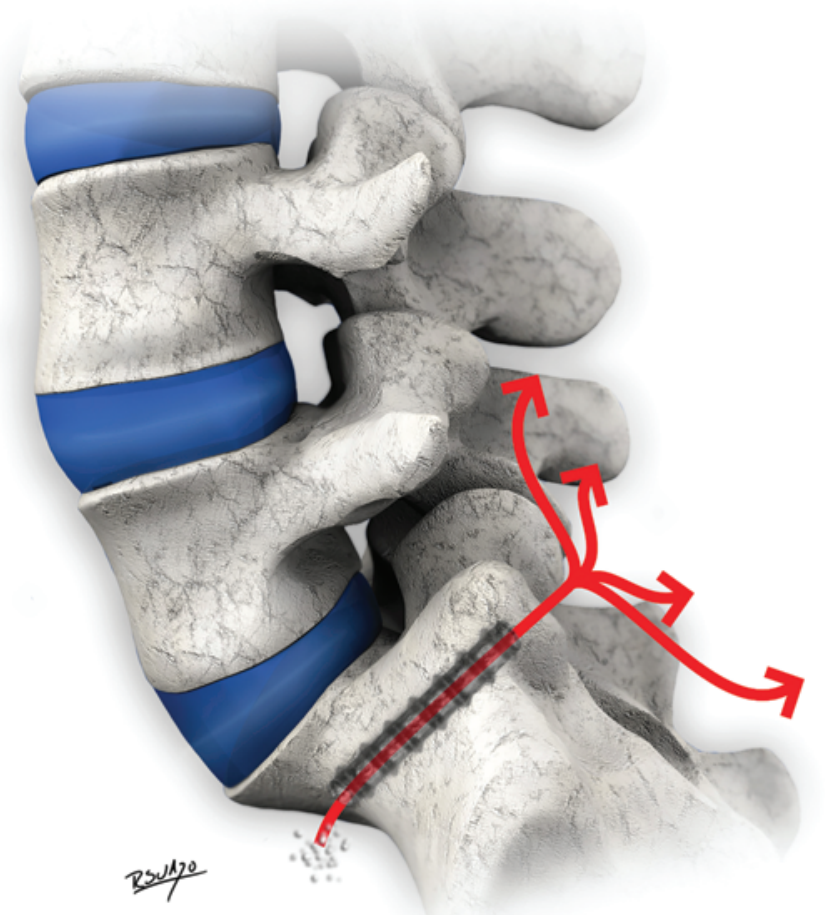

FIG. 4. A 3D reconstruction of the conduit produced by removal of spinal instrumentation. Here we demonstrate the ability of microbes and debris to pass back and forth between the posterior and anterior aspects of the spine. Copyright Roberto Suazo. Published with permission.

the beginning of this patient's presacral collection can be noted. Although subtle, this abnormal presacral enhancement almost certainly progressed into the larger abscess that was eventually drained anteriorly.

Our patient continued to present with recurrent infections despite the treatment protocol, which has been documented in the literature as a means to eradicate SSI. Of the 17 patients described by Levi et al., 15 required just $1 \mathrm{I} \& \mathrm{D}$, whereas the other 2 patients required a second I $\&$ D. At the 8-month follow-up visit there were no recurrent infections. ${ }^{14}$ Of the 21 patients with SSIs described by Chaichana et al., only 1 required 2 washouts to eradicate the infection, whereas all other patients required only 1 washout, and at follow-up no patients had signs of recurrent infection. ${ }^{3}$ In contrast to what has been reported in the literature, our patient presented with 7 recurrent infections requiring 8 I \& Ds and numerous rounds of antibiotics over a 5-year period, including indefinite suppressive therapy. A major factor in the difficulty in treating our patient's recurrent infections was the presence of simultaneous presacral and paraspinal abscesses that communicated via the bicortical sacral screw tracts (Fig. 4).

\section{Conclusions}

This patient presented with multiple recurrences of SSI after lumbar spinal instrumentation and required a total of 8 I \& Ds over a 5-year period due to an unrecognized presacral abscess following placement of bicortical sacral screws. The importance of this case is to highlight a mech- 
anism of seeding to the presacral space after removal of spinal instrumentation, a process not previously described in the literature. Although biomechanical advantages are conferred with the use of bicortical sacral screws, careful surveillance, with attention to the presacral space, should be paid in infections requiring removal of hardware.

\section{References}

1. Akesen B, Wu C, Mehbod AA, Transfeldt EE: Biomechanical evaluation of paracoccygeal transsacral fixation. J Spinal Disord Tech 21:39-44, 2008

2. Barrey C, Launay O, Freitas E, Michel F, Laurent F, Chidiac $\mathrm{C}$, et al: The follow-up of patients with postoperative infection of the spine. Eur J Orthop Surg Traumatol 23 (Suppl 1):S29-S34, 2013

3. Chaichana KL, Bydon M, Santiago-Dieppa DR, Hwang L, McLoughlin G, Sciubba DM: Risk of infection following posterior lumbar fusion for degenerative spine disease in 817 consecutive cases. J Neurosurg Spine 20:45-42, 2014

4. Cizik AM, Lee MJ, Martin BI, Bransford RJ, Bellabarba C, Chapman JR, et al: Using the spine surgical invasiveness index to identify risk of surgical site infection: a multivariate analysis. J Bone Joint Surg Am 94:335-342, 2012

5. Ergur I, Akcali O, Kiray A, Kosay C, Tayefi H: Neurovascular risks of sacral screws with bicortical purchase: an anatomical study. Eur Spine J 16:1519-1523, 2007

6. Fang XT, Wood KB: Management of postoperative instrumented spinal wound infection. Chin Med J (Engl) 126:3817-3821, 2013

7. Gelalis ID, Arnaoutoglou CM, Politis AN, Batzaleksis NA, Katonis PG, Xenakis TA: Bacterial wound contamination during simple and complex spinal procedures. A prospective clinical study. Spine J 11:1042-1048, 2011

8. Gerometta A, Rodriguez Olaverri JC, Bitan F: Infections in spinal instrumentation. Int Orthop 36:457-464, 2012

9. Hedequist D, Haugen A, Hresko T, Emans J: Failure of attempted implant retention in spinal deformity delayed surgical site infections. Spine (Phila Pa 1976) 34:60-64, 2009

10. Hegde V, Meredith DS, Kepler CK, Huang RC: Management of postoperative spinal infections. World J Orthop 3:182189, 2012

11. Jutte PC, Castelein RM: Complications of pedicle screws in lumbar and lumbosacral fusions in 105 consecutive primary operations. Eur Spine J 11:594-598, 2002

12. Kasliwal MK, Tan LA, Traynelis VC: Infection with spinal instrumentation: Review of pathogenesis, diagnosis, prevention, and management. Surg Neurol Int 4 (Suppl 5):S392S403, 2013
13. Leong JC, Lu WW, Zheng Y, Zhu Q, Zhong S: Comparison of the strengths of lumbosacral fixation achieved with techniques using one and two triangulated sacral screws. Spine (Phila Pa 1976) 23:2289-2294, 1998

14. Levi ADO, Dickman CA, Sonntag VKH: Management of postoperative infections after spinal instrumentation. J Neurosurg 86:975-980, 1997

15. Meredith DS, Kepler CK, Huang RC, Brause BD, BoachieAdjei O: Postoperative infections of the lumbar spine: presentation and management. Int Orthop 36:439-444, 2012

16. O’Toole JE, Eichholz KM, Fessler RG: Surgical site infection rates after minimally invasive spinal surgery. J Neurosurg Spine 11:471-476, 2009

17. Pull ter Gunne AF, Cohen DB: Incidence, prevalence, and analysis of risk factors for surgical site infection following adult spinal surgery. Spine (Phila Pa 1976) 34:1422-1428, 2009

18. Pull ter Gunne AF, Mohamed AS, Skolasky RL, van Laarhoven CJ, Cohen DB: The presentation, incidence, etiology, and treatment of surgical site infections after spinal surgery. Spine (Phila Pa 1976) 35:1323-1328, 2010

19. Quaile A: Infections associated with spinal implants. Int Orthop 36:451-456, 2012

20. Rayes M, Colen CB, Bahgat DA, Higashida T, Guthikonda M, Rengachary S, et al: Safety of instrumentation in patients with spinal infection. J Neurosurg Spine 12:647-659, 2010

21. von Strempel A, Trenkmann S, Krönauer I, Kirsch L, Sukopp C: The stability of bone screws in the os sacrum. Eur Spine J 7:313-320, 1998

\section{Disclosures}

Dr. Levi receives a teaching honorarium from Medtronic Sofamor Danek.

\section{Author Contributions}

Conception and design: all authors. Acquisition of data: all authors. Analysis and interpretation of data: all authors. Drafting the article: all authors. Critically revising the article: Levi, Bloom. Reviewed submitted version of manuscript: Levi, Burks. Approved the final version of the manuscript on behalf of all authors: Levi. Administrative/technical/material support: Burks.

\section{Correspondence}

Allan D. Levi, Department of Neurological Surgery, University of Miami Miller School of Medicine, Lois Pope Life Center, 1095 N.W. 14th Terrace (D4-6), Miami, FL 33136. email: alevi@med.miami.edu. 